\title{
Self-Assembly of Okadaic Acid as a Pathway to the Cell
}

Antonio Hernández Daranas, ${ }^{\dagger, \ddagger}$ Patricia G. Cruz, ${ }^{\dagger}$ Alberto Hernández Creus, ${ }^{* \S}$ Manuel Norte, ${ }^{\dagger}$ and José J. Fernández ${ }^{*, \dagger}$

Instituto Universitario de Bio-Orgánica “Antonio González”, Astrofísico Francisco Sánchez 2,

Universidad de La Laguna, 38206 La Laguna, Tenerife, Spain, Departamento de Química-

Física and Departamento de Ingeniería Química y Tecnología Farmacéutica, Universidad de La Laguna, 38071 La Laguna, Tenerife, Spain

\section{Supporting Information}

STM images (constant current mode) were taken with a Nanoscope IIE (Digital Instrument, Santa Barbara, CA) using both, commercial and electrochemically etched Pt-Ir tips. All samples were thoroughly rinsed with water, methanol and dried and under $\mathrm{N}_{2}$ flow for several hours before imaging. All images were taken in air at room temperature. Typical tunneling current and applied bias voltages to obtain good quality images were 0.2-0.4 nA and 0.3-0.6 $\mathrm{V}$ respectively at scan rates ranging $0.5-2 \mathrm{~Hz}$. The STM images shown here constitute representative results. Au plates (arrandee ${ }^{\mathrm{TM}}$ ) were used as substrate. These plates after a flame annealing consist of micrometer-sized $\mathrm{Au}(111)$ preferred oriented crystals with atomically smooth triangular terraces separated by monatomic steps in height. The height of these steps (2.4-2.6 $\AA$ ) was used to calibrate the piezotube of the STM in the $z$ direction. 

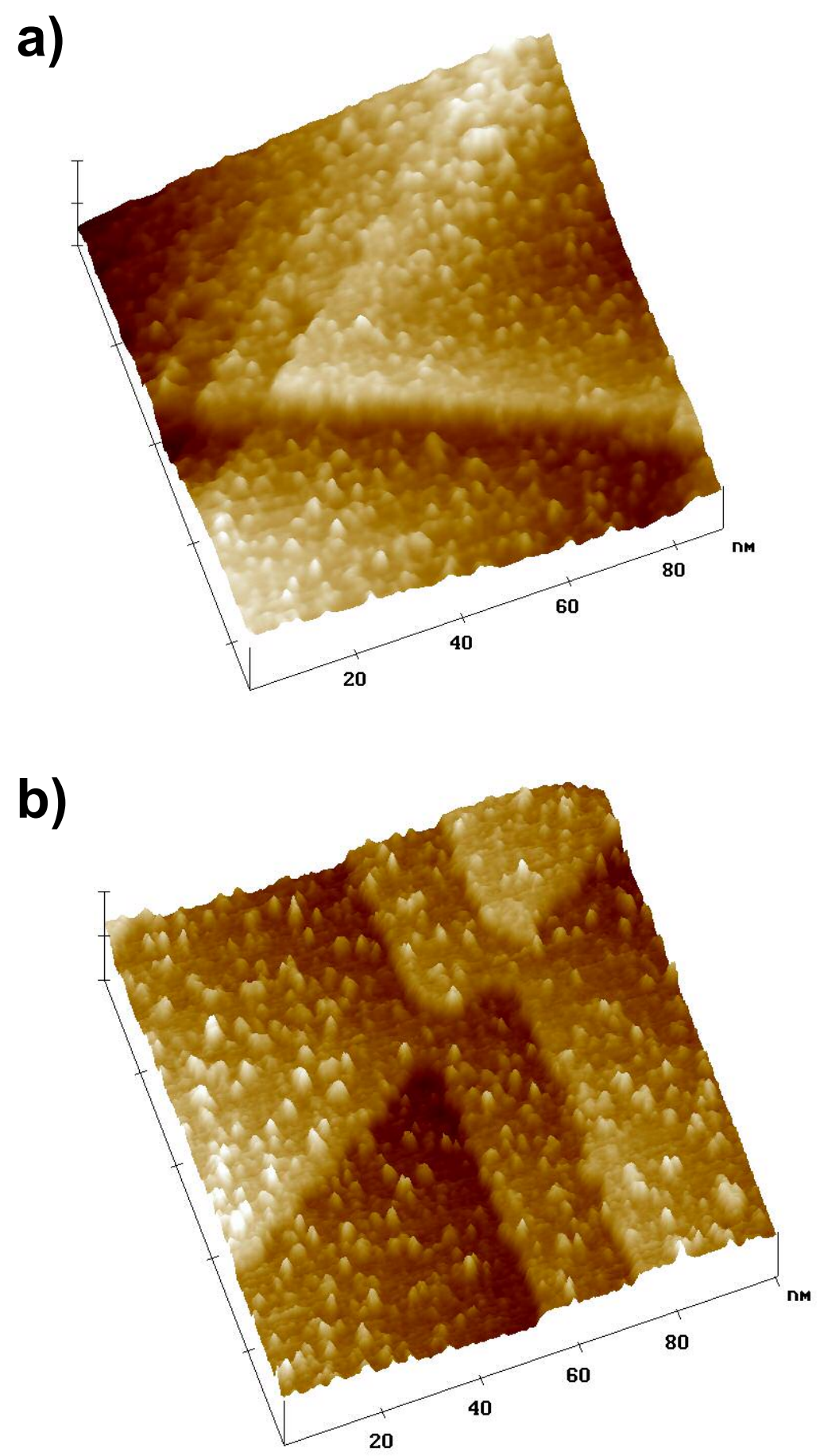

Figure 1

Figures 1a) and 1b) show representative Au(111) terraces observed after dipping the gold plates for $24 \mathrm{hr}$ in a $0.5 \mathrm{mM}$ okadaic acid solution in methanol. As can be seen, okadaic acid adsorption takes place spontaneously, covering the entire gold surface and can be easily detected by STM. The height of these particles $(2.5 \AA)$ is similar or slightly greater than that of a monatomic Au step. The $z$ scale is $1 \mathrm{~nm} / \mathrm{div}$. 
Adsorption of okadaic acid molecules onto the gold surface is proportional to both the dipping time and okadaic acid concentration. Therefore, in order to optimize the quality of STM images we conducted several experiments varying both parameters. Best results where found by dipping the gold plate for two hours into a $0.5 \mathrm{mM}$ okadaic acid solution.
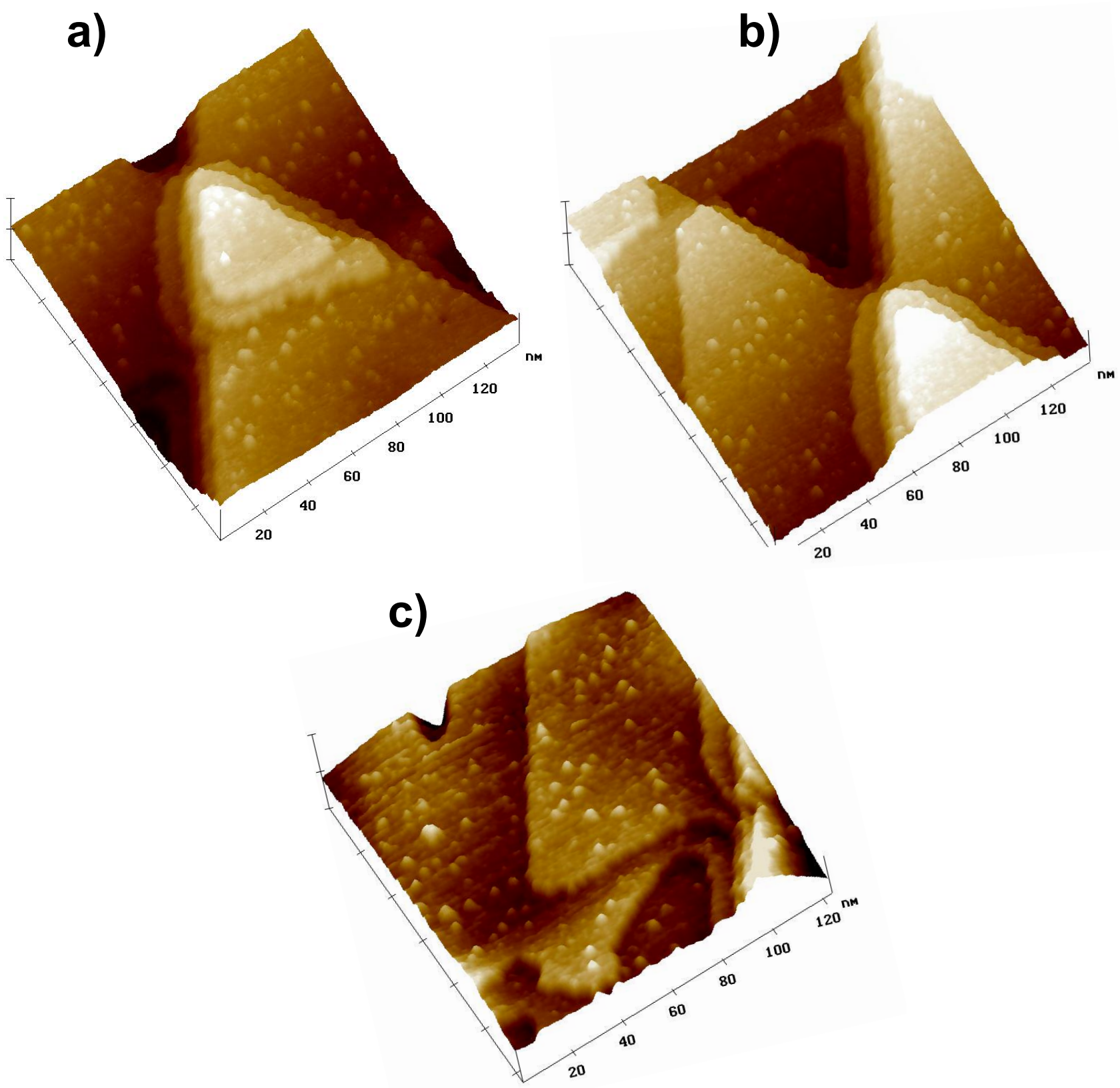

\section{Figure 2}

Figures 2 a)-c) show some gold terraces covered by okadaic acid molecules. It should be noted that although most of them are $22-25 \AA$ in size, a number of bigger particles around $40-45 \AA$ could be found. This can be clearly observed in Figure 3 and its corresponding cross section. Taking into account the geometry of okadaic acid molecules, this result means that particles detected by STM correspond to monomers and dimers of okadaic acid molecules. The histogram of size distribution (Figure 8) reflects this fact as well as that the major sizes detected are distributed around 20-25 and $40-45 \AA$. The $z$ scale in all Figure 2 is $1 \mathrm{~nm} / \mathrm{div}$. 
a)

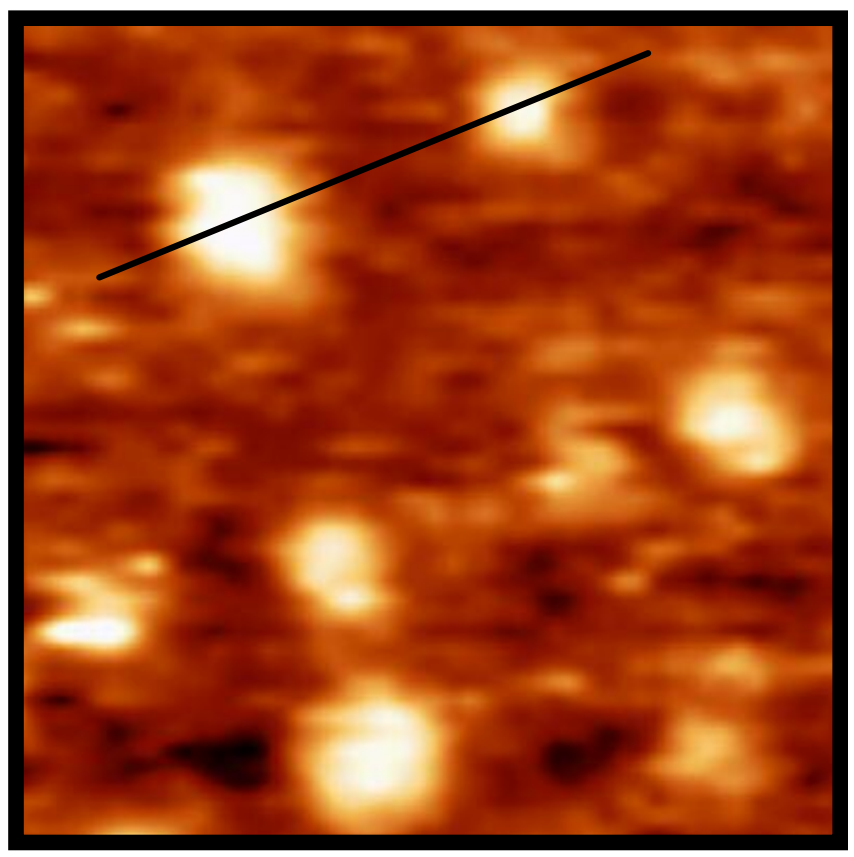

$20 \mathrm{~nm} \times 20 \mathrm{~nm}$

b)

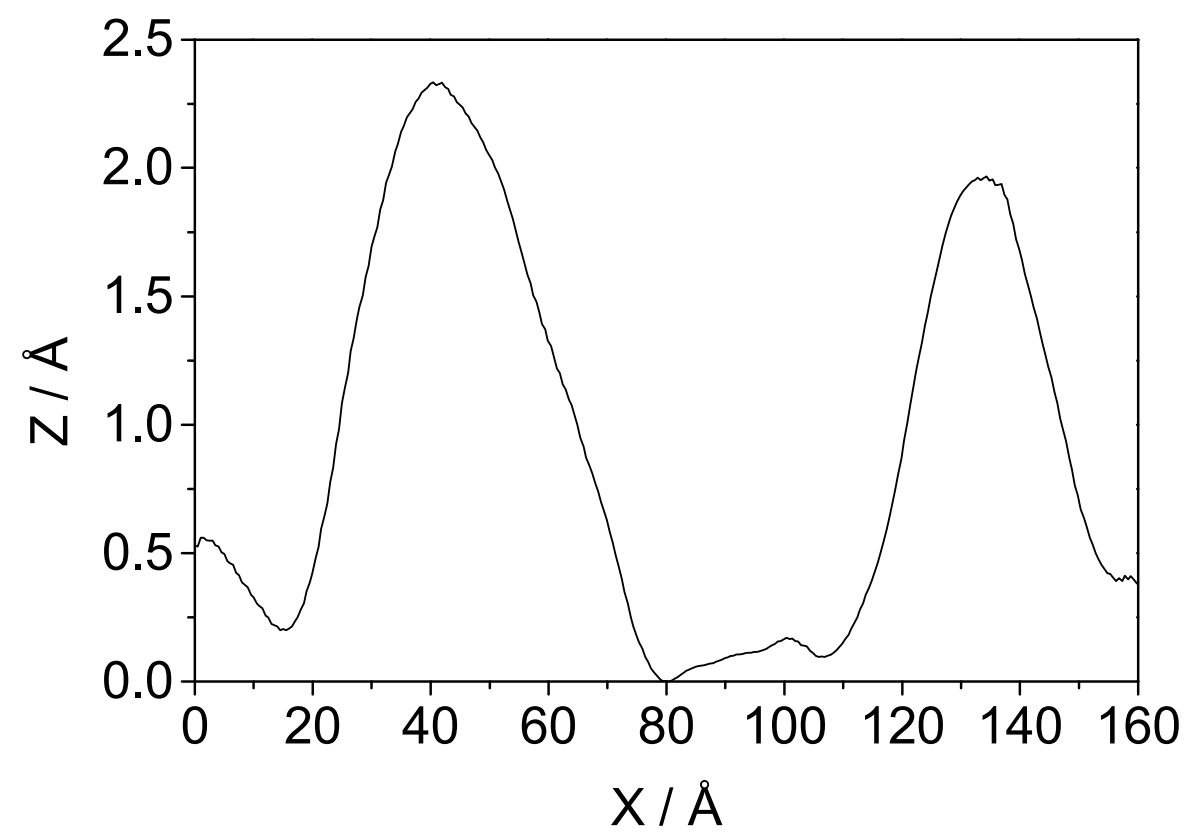

Figure 3

Figures 3 a) and b) show STM image (20 nm $\times 20 \mathrm{~nm}$ ), and its corresponding cross sections and showing monomeric (20-25 $\AA$ ) and dimeric (40-45 $\AA$ ) forms of okadaic acid. 
The experiment shown in Figure 2 was repeated afterwards in a solution containing additionally $5 \mathrm{mM}$ in $\mathrm{KCl}$. As can be seen in Figures 4 a)-c) and the corresponding cross section (Figure 5) the gold surface appeared covered by okadaic acid molecules, many of them around $40-45 \AA$ in size and $2.5 \AA$ in height. The surface density is around 5000 molecules per squared micron. Under these experimental conditions, the histogram of sizes distribution shows an increment in the average particle size. The $z$ scale all Figures 4 is $1 \mathrm{~nm} /$ div.
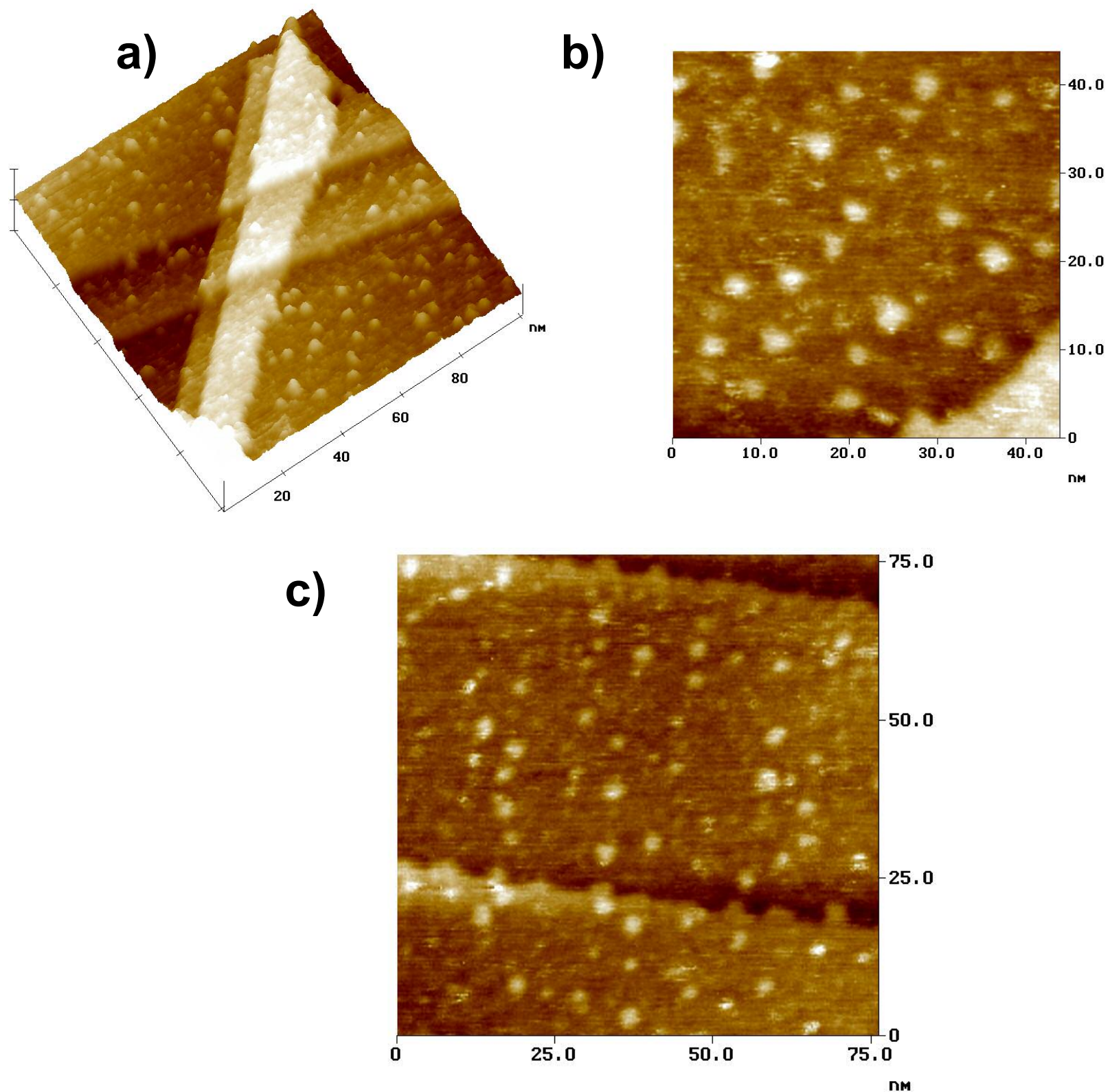

Figure 4

Figures 4 a)-c) show some gold terraces covered by okadaic acid molecules in dimeric form. 
a)

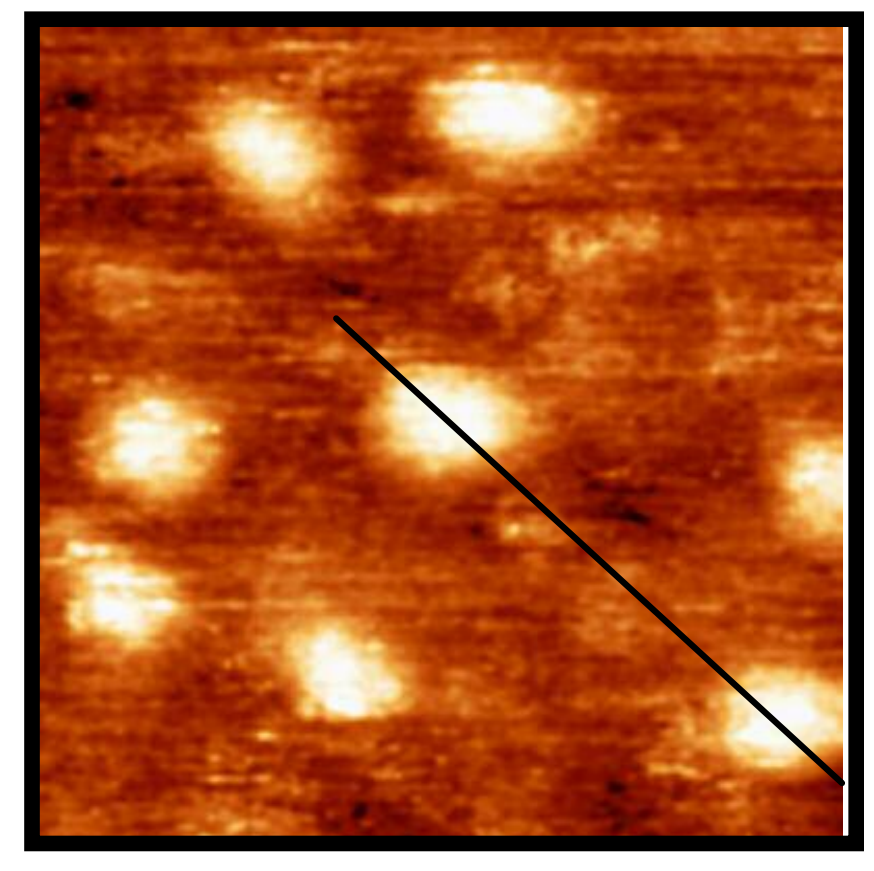

$20 \mathrm{~nm} \times 20 \mathrm{~nm}$

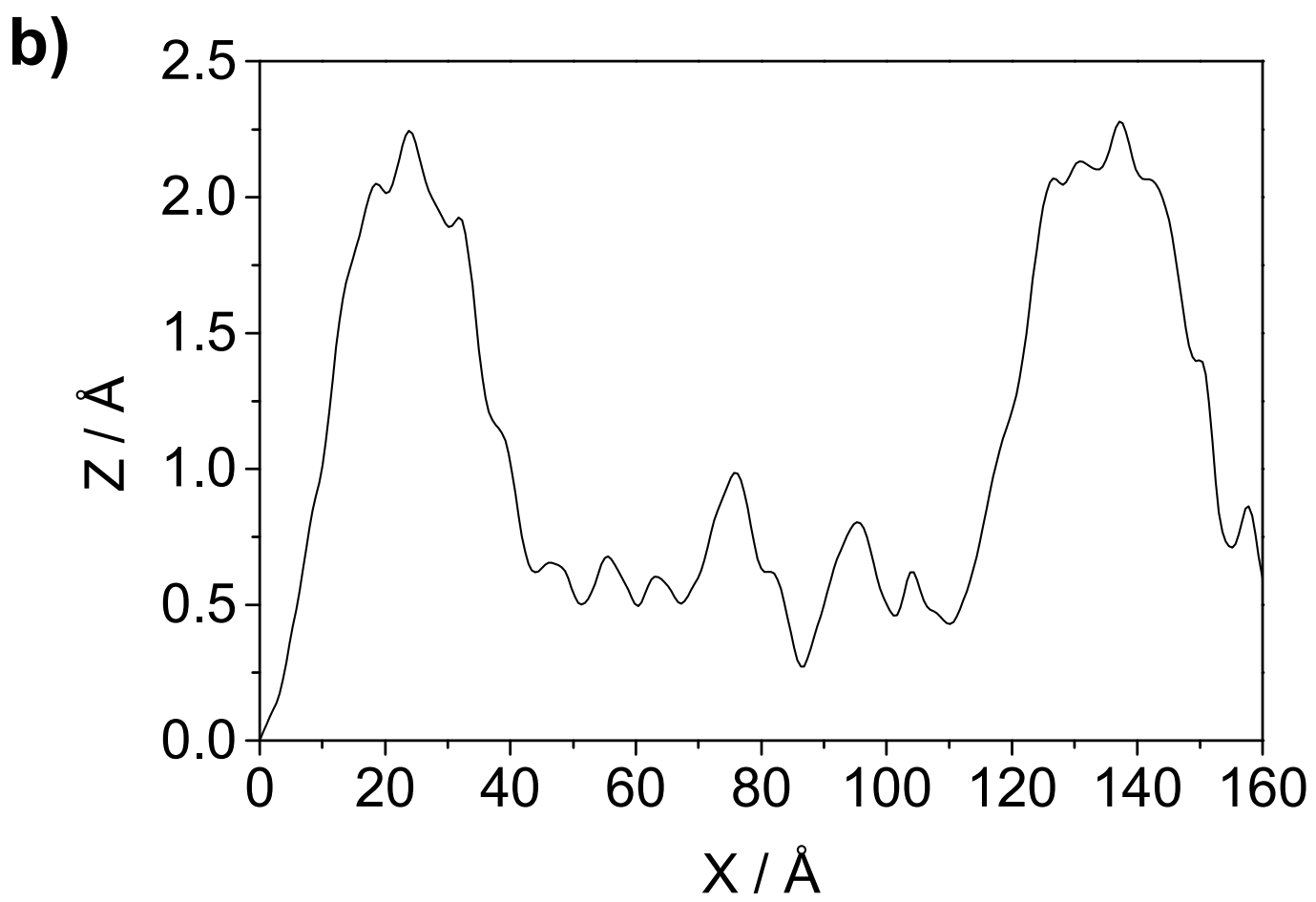

Figure 5

Figures 5 a) and b) show STM image (20 nm $\times 20 \mathrm{~nm}$ ), and its corresponding cross sections showing dimeric (40-45 $\AA$ ) forms of okadaic acid. 


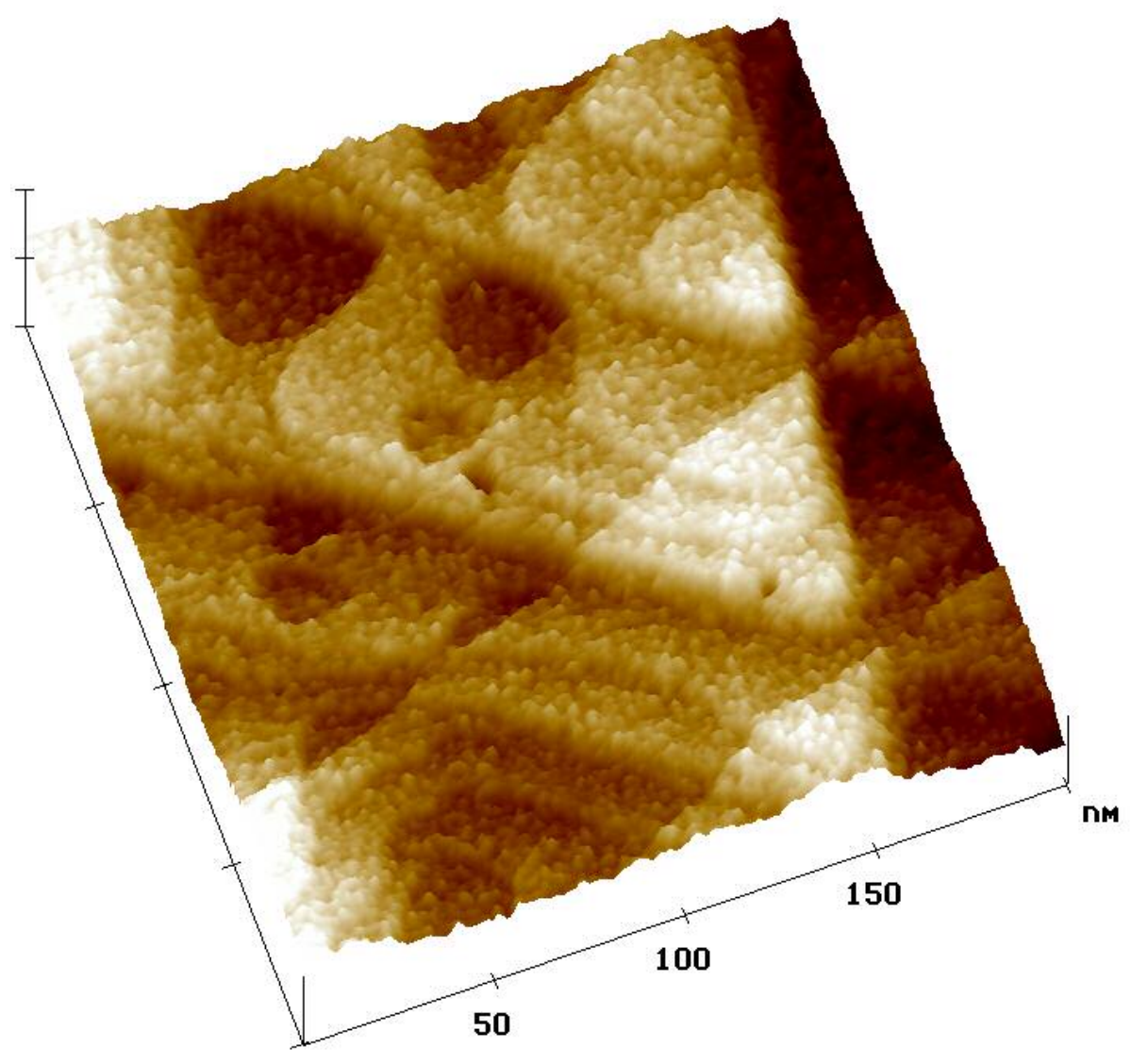

Figure 6

Figure 6 shows STM image obtained after a potential of $-90 \mathrm{mV}$ was directly aplied to an $\mathrm{Au}(111)$ plate dipped in an okadaic acid $(0.5 \mathrm{mM})$ and $\mathrm{KCl}(5 \mathrm{mM})$ methanol solution for $2 \mathrm{~h}$. Note that the gold surface appears totally covered by okadaic acid molecules. The $z$ scale is $2 \mathrm{~nm} /$ div. 


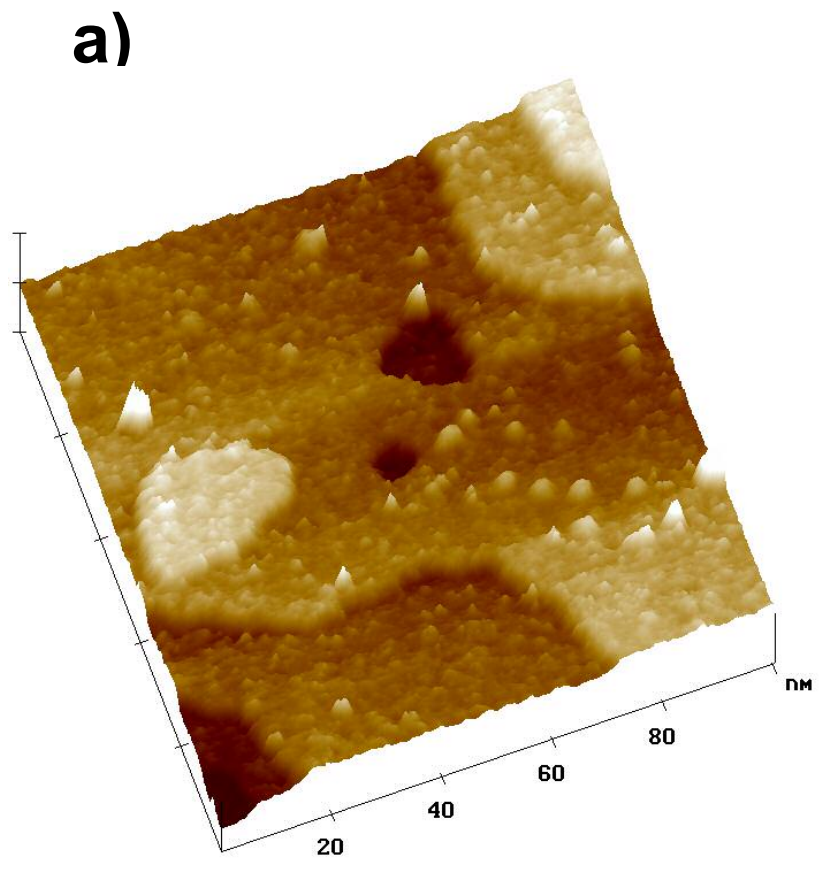

b)

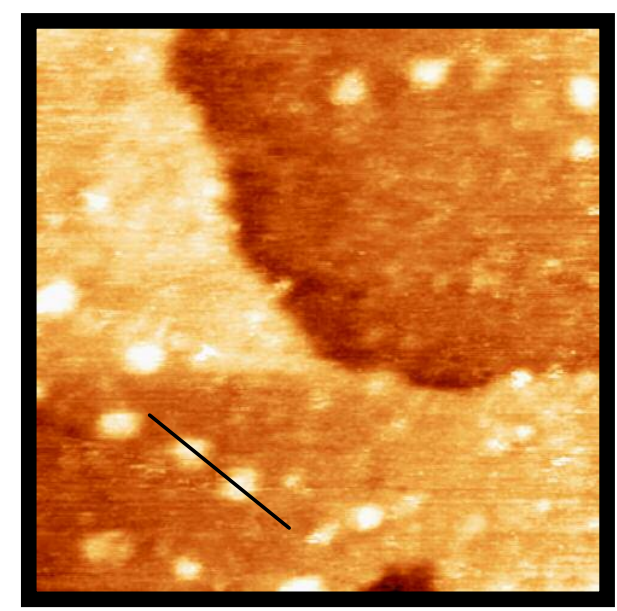

$50 \mathrm{~nm} \times 50 \mathrm{~nm}$

c)

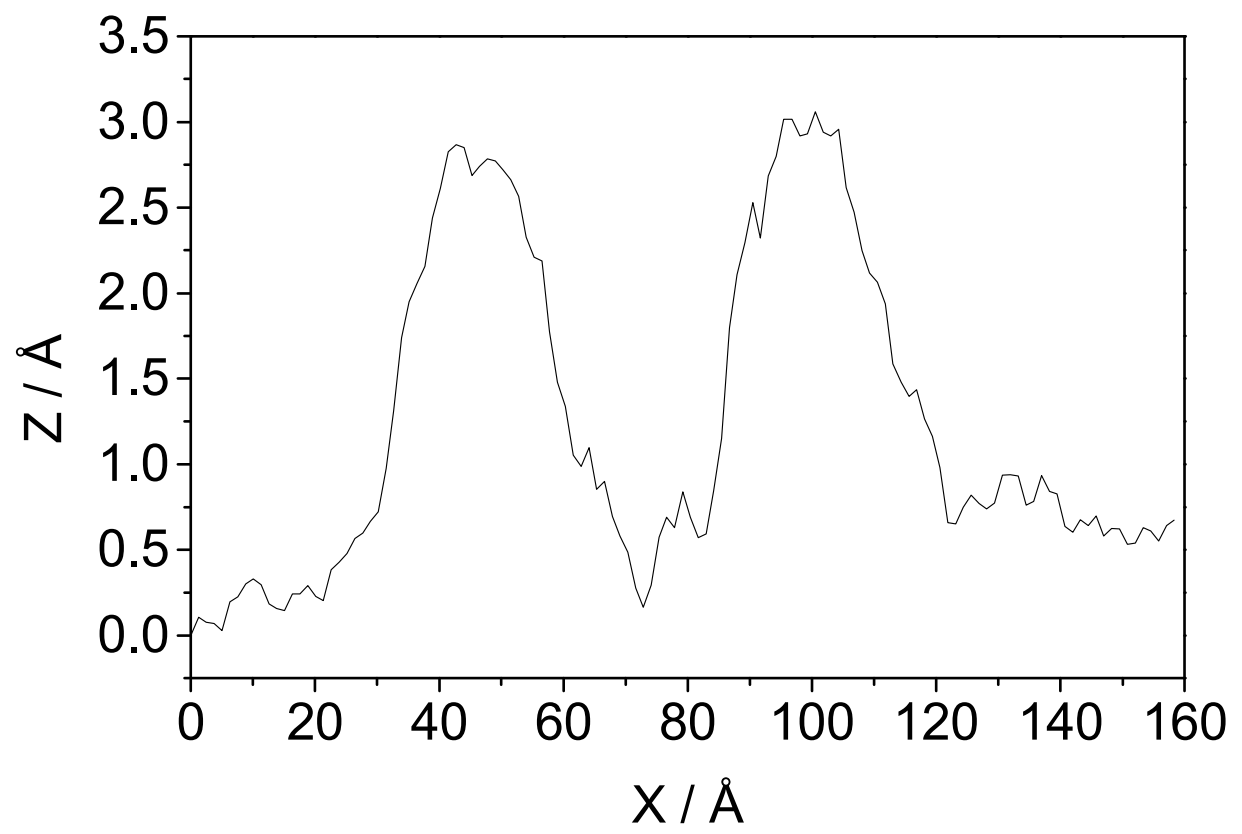

Figure 7

Figures 7 a)-c) show the gold plate surface and the corresponding cross section obtained when the experiment was made using a lipid bilayer. The $z$ scale is $2 \mathrm{~nm} / \mathrm{div}$. 
a)
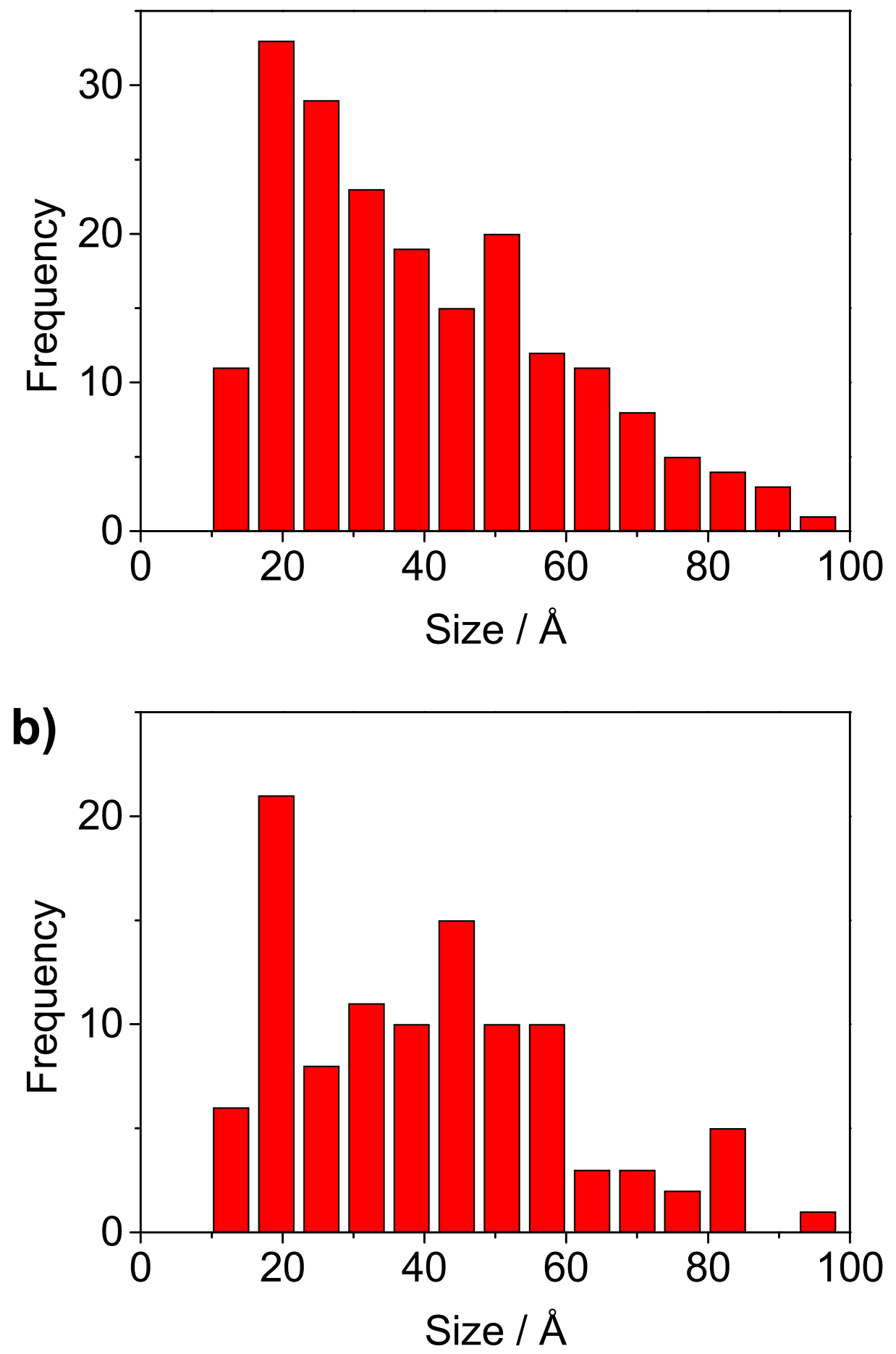

Figure 8

Figures 8 a) and b) show the histograms of size distribution of the particles as measured after a gold plate was dipped for $2 \mathrm{~h}$ in a) $0.5 \mathrm{mM}$ solution of okadaic acid in methanol, and b) $0.5 \mathrm{mM}$ and $\mathrm{KCl} 5 \mathrm{mM}$ solution o okadaic acid in methanol. 